\title{
One-piece Orbitozygomatic Craniotomy for Resection of Rathke's Cleft Cyst: Operative Video
}

\author{
Rimal H. Dossani ${ }^{1}$ Devi P. Patra ${ }^{1}$ Christopher Storey ${ }^{1}$ Piyush Kalakoti ${ }^{1}$ Hai Sun ${ }^{1}$ \\ ${ }^{1}$ Department of Neurosurgery, Louisiana State University Health \\ Sciences Center, Shreveport, Louisiana, United States \\ Address for correspondence Rimal H. Dossani, MD, Department of \\ Neurosurgery, Louisiana State University Health Sciences Center, \\ 1501 Kings Highway, Shreveport, LA 71130-3932, United States \\ J Neurol Surg B 2018;79(suppl S2):S211-S212. \\ (e-mail: rhanif@lsuhsc.edu).
}

\begin{abstract}
Keywords

- orbitozygomatic craniotomy

- Rathke's cleft cyst

- suprasellar

- pterional

- optic nerves

- MRI

- skull base

- operative technique

Objective The video stars orbitozygomatic resection of Rathke's cleft cyst with suprasellar extension in a 37-year-old male patient presenting with severe headaches and bitemporal hemianopia. Clinical and radiological characteristics along with surgical technique (positioning, bony opening, surgical dissection and debulking, closure), histopathology, and postoperative course are described.

Methods Preoperative MRI demonstrated a noncontrast-enhancing cystic lesion in the sella with suprasellar extension causing compression of both optic nerves. A onepiece orbitozygomatic craniotomy was performed. The tumor was encountered in the interoptic space. First, the cyst was decompressed and fluid appearing like motor oil was aspirated. Both optic nerves were decompressed and dissected free from the cyst wall. Intraoperatively, the most challenging aspect was separating the tumor from surrounding vascular structures, including bilateral A1 arteries and the left carotid bifurcation. A combination of sharp and blunt dissection was utilized to free the tumor from adhesions to critical neurovascular structures. Once freed, the suprasellar aspect of the tumor was mobilized into the operative cavity and debulked. Finally, the sellar component of the tumor was removed all the way down to the sellar floor. Postoperative MRI demonstrated decompressed bilateral optic nerves with an intact pituitary stalk with preservation of normal pituitary gland. Histopathology identified pathognomonic features consistent with diagnosis of Rathke's cleft cyst, including flattened ciliated epithelium and presence of Rathke's cleft remnants.

Results Postoperatively, bilateral improvement in vision was noted with transient diabetes insipidus. Patient was discharged home on postoperative day 4.

Conclusion A one-piece orbitozygomatic craniotomy is an effective and safe strategy for resection of Rathke's cleft cysts with suprasellar extension.

The link to the video can be found at: https://youtu.be/-Yqtcd2gLSs.
\end{abstract}

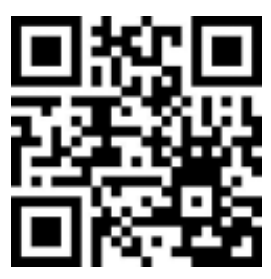

received

October 16, 2017

accepted

November 29, 2017

published online

January 11, 2018

www.thieme.com/skullbasevideos

www.thieme.com/jnlsbvideos

DOI https://doi.org/

10.1055/s-0037-1620277.

ISSN 2193-6331.
(C) 2018 Georg Thieme Verlag KG Stuttgart · New York
License terms

(ㄷ) (i) $\ominus$ (\$) 
Conflict of Interest

None.

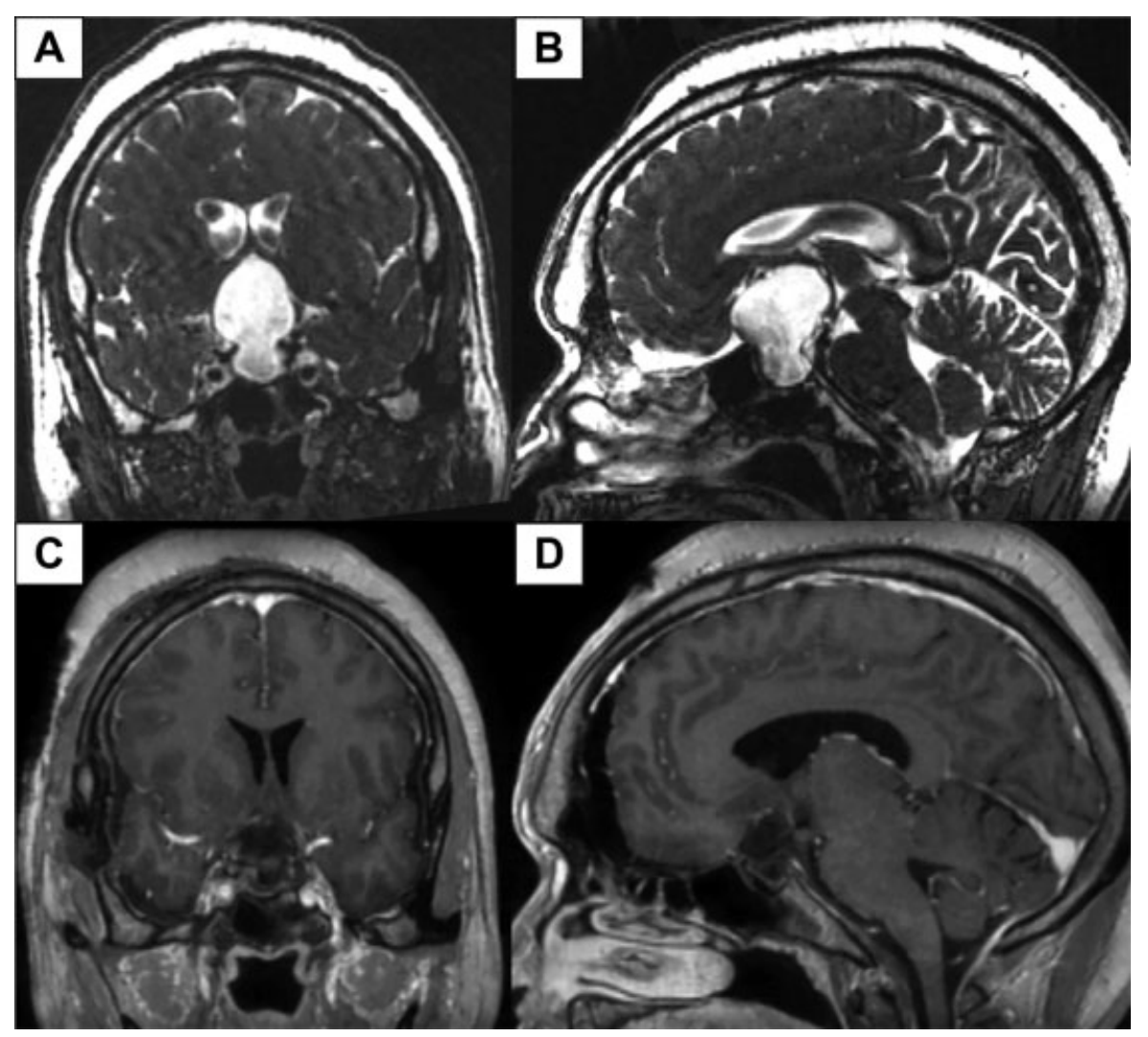

Fig. 1 MR imaging (T2 weighted) demonstrating noncontrast-enhancing cystic lesion in the sella with suprasellar extension causing compression of both optic nerves in the (A) coronal and (B) sagittal sections. (C-D) Postoperative MR imaging demonstrating adequate debulking in the coronal and sagittal planes. MR, magnetic resonance.

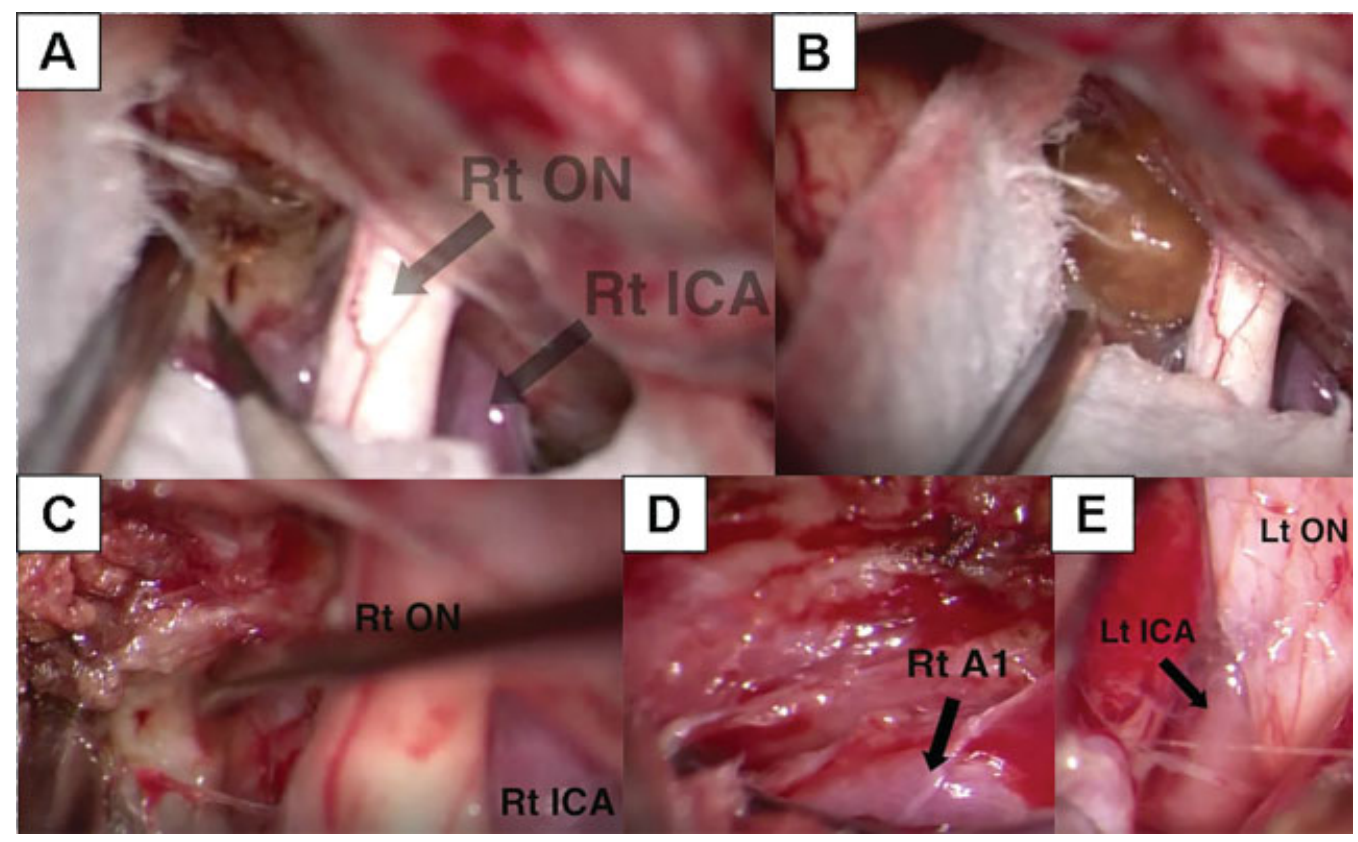

Fig. 2 Intraoperative images demonstrating (A) cyst decompression using sharp dissection (puncture). The panel also depicts the relation of the cyst with respect to the right optic nerve (black arrow) and internal carotid artery (black arrow). (B) Release of cystic contents (thick, red, motor oil-like fluid) following cystic decompression. (C) Decompression of right optic nerve from the cyst wall followed by (D) separation of the A1 branch of the right anterior cerebral artery (black arrow) from the tumor capsule. (E) Cyst wall being separated from neurovascular adhesions including left optic nerve and the left internal carotid artery (black arrow). ICA, internal carotid artery; ON, optic nerve. 